\title{
Surgical retrieval of embolized atrial septum defect closure device from right pulmonary vein
}

\author{
Fei Zhao ${ }^{1}$ and Yongjun Qian ${ }^{2}$ \\ ${ }^{1}$ Sichuan University West China Hospital Department of Radiology \\ ${ }^{2}$ Sichuan University West China Hospital
}

February 8, 2022

\begin{abstract}
A 69-year-old woman presented to the emergency department for symptoms of acute right heart failure including progressive exertional dyspnea and limbs weakness. Physical examination showed slight cyanosis and cold limbs. She underwent percutaneous transcatheter closure of a secundum ASD through femoral vein eight months before. On further investigation, transthoracic echocardiography (TTE) revealed severe tricuspid regurgitation and the migrated ASD device (Figure 1), and the embolization seemed to be located near the left ventricular inflow tract. Computed tomography (CT) and three-dimensional reconstruction confirmed the left-side migration of the device, and revealed obstruction of RPV caused by the embolization (Figure 2).
\end{abstract}

\section{Title Page}

Surgical retrieval of embolized atrial septum defect closure device from right pulmonary vein Fei Zhao ${ }^{1}$, Yongjun Qian²\#

${ }^{1}$ Department of Radiology, West China Hospital, Sichuan University, Guoxuexiang 37th, 610041 Chengdu, Sichuan, P.R. China.

${ }^{2}$ Department of Cardiovascular Surgery, West China Hospital, Sichuan University, Guoxuexiang 37th, 610041 Chengdu, Sichuan, P.R. China.

\# Correspondence: Yongjun Qian, No. 37 GuoXue Xiang, Chengdu, Sichuan, 610041, People's Republic of China; fax: + 8628 85422897; e-mail: qianyongjun@scu.edu.cn .

Surgical retrieval of embolized atrial septum defect closure device from right pulmonary vein Fei Zhao ${ }^{1}$, Yongjun Qian ${ }^{2 \#}$

${ }^{1}$ Department of Radiology, West China Hospital, Sichuan University, Guoxuexiang 37th, 610041 Chengdu, Sichuan, P.R. China.

${ }^{2}$ Department of Cardiovascular Surgery, West China Hospital, Sichuan University, Guoxuexiang 37th, 610041 Chengdu, Sichuan, P.R. China.

\# Correspondence: Yongjun Qian, No. 37 GuoXue Xiang, Chengdu, Sichuan, 610041, People's Republic of China; fax: + 8628 85422897; e-mail: qianyongjun@scu.edu.cn .

A 69-year-old woman presented to the emergency department for symptoms of acute right heart failure including progressive exertional dyspnea and limbs weakness. Physical examination showed slight cyanosis and cold limbs. She underwent percutaneous transcatheter closure of a secundum ASD through femoral vein eight months before. On further investigation, transthoracic echocardiography (TTE) revealed severe 
tricuspid regurgitation and the migrated ASD device (Figure 1), and the embolization seemed to be located near the left ventricular inflow tract. Computed tomography $(\mathrm{CT})$ and three-dimensional reconstruction confirmed the left-side migration of the device, and revealed obstruction of RPV caused by the embolization (Figure 2). Given the clinical features and radiological findings, the diagnosis of ASD closure device migration was decided, and symptoms of heart failure were caused by RPV obstruction. In consideration of eight months after percutaneous closure, a safe surgical retrieval procedure reported by our center before was performed [1]. Standard median sternotomy and cardiopulmonary bypass were performed first, access was via right atrium. Then removed the endothelialized tissue around the device, and the "knot" of device was held up and cut off, the fine wires were spread out and retrieved one by one. Finally, the device was easily retrieved when only two wires remained, and the ASD was closed surgically.

Postoperative vital signs were stable and the patient was discharged for home without symptoms of right heart failure. At the follow up one month later, TTE revealed no significant residual leaks at the ASD level, and no dyspnea or weakness was found.

Many complications such as deice embolization, erosion, bleeding, arrhythmias, infection, air embolism and thromboembolism have been reported in previous studies [2,3], embolization has the highest incidence rate among all complications. The major time of embolization is reported in the first $24 \mathrm{~h}$, and the most common sites are the main or branched pulmonary artery and atrioventricular valve, rarely migrate to the left side.However, several weeks or months after deployment, because of endothelialization, device is firmly fixed in the tissue, there is little chance for device to migrate. On the other hand, once these devices of endothelial happen to migrate, retrieving the embolization percutaneously is hard and dangerous. Surgical retrieval could be selected and done with less risks and better results when failing or hard to retrieve percutaneously. Techniques such as direct retrieval and using two forceps to fold the device have been reported [4]. In this case, we performed a safe procedure to retrieve the migrated device, as our center has used this procedure to retrieve migrated ventricular septal defect closure device [1], which could minimize valve and conduction system complications.

\section{DECLARATION OF PATIENT CONSENT}

The authors certify that they have obtained all appropriate patient consent forms. The patients understand that their names and initials will not be published and due efforts will be made to conceal their identity, but anonymity cannot be guaranteed.

\section{CONFLICT OF INTERESTS}

The authors declare that there are no conflict of interests.

\section{REFERENCES}

1. Lin G, Su Y, Qian YJ. Safe Surgical Retrieval of Endothelialized Ventricular Septal Defect Closure Device. Heart Surg Forum. 2020 May 6;23(3):E285-E286.

2. Karampinis I, Pfleger S, Nowak K. Extracardial migration of an atrial septal defect occluder device. J Invasive Cardiol. 2013 Dec;25(12):E219-20.

3. Yang MC, Wu JR. Recent review of transcatheter closure of atrial septal defect. Kaohsiung J Med Sci. $2018 \mathrm{Jul} ; 34(7): 363-369$.

4. Yates MT, Anderson DR. Safe Surgical Retrieval of Embolized Atrial Septal Defect Closure Device. Ann Thorac Surg. 2017 Feb;103(2):e213-e214.

\section{Figures}

Figure 1. Transthoracic echocardiography revealed tricuspid regurgitation and malposition of the device. $(\mathrm{RA}=$ right atrium, $\mathrm{RV}=$ right ventricle, $\mathrm{TV}=$ tricuspid valve, $\mathrm{D}=$ device $)$ 
Figure 2. Three-dimensional reconstruction demonstrated right pulmonary vein obstruction caused by migrated device. (The green object is device; $\mathrm{D}=$ device, $\mathrm{RPV}=$ right pulmonary vein)
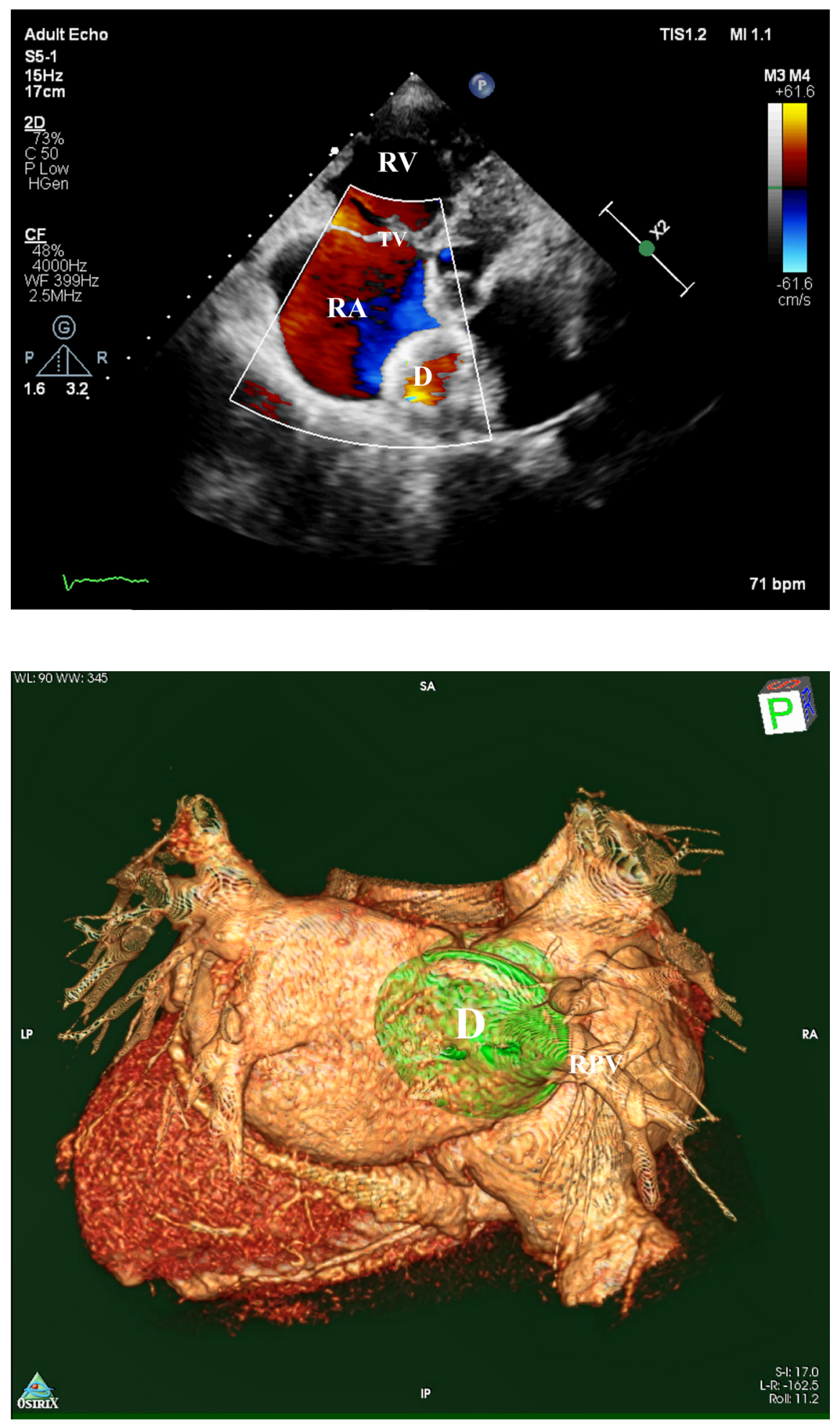